\title{
THE TEICHMÜLLER SPACE OF A COUNTABLE SET OF POINTS ON A RIEMANN SURFACE
}

\author{
EGE FUJIKAWA AND MASAHIKO TANIGUCHI
}

\begin{abstract}
We introduce the quasiconformal deformation space of an ordered countable set of an infinite number of points on a Riemann surface and give certain conditions under which it admits a complex structure via Teichmüller spaces of associated subsurfaces with the complement of the set of points. In a similar fashion, we give another definition of the quasiconformal deformation space of a finitely generated Kleinian group.
\end{abstract}

\section{INTRODUCTION}

The Teichmüller space is the deformation space of complex structures on a topological surface and it is well known that it has a standard complex structure. However, in the infancy of the theory of Teichmüller spaces, it was often confused with the deformation space of conformal classes of the point configurations on a Riemann surface. A typical example was the space of affine classes of the configurations of three finite critical points of Weierstrass's $\wp$ function, which was called the moduli parameter of elliptic functions. Also, Grötzsch, a pioneer of the Teichmüller theory, considered the deformation space of Möbius classes of the configurations of four points on the Riemann sphere $\widehat{\mathbb{C}}$.

Actually, even for the configurations of a finite number of points on a Riemann surface, there is some difference between the deformation space of their conformal classes and the Teichmüller space of the complement of the points. In this case, the former turns out to be a quotient complex manifold of the latter by a certain subgroup of the Teichmüller modular group, and hence we can regard the deformation space, or so-called the moduli space, of the point configurations as a complex manifold. From the viewpoint of holomorphic dynamics induced by the iteration of a holomorphic map, the countable set of periodic points plays an important role. To consider this object, we will be faced with the necessity of introducing the deformation space of conformal classes of the configurations, even for a countably infinite number of points which does not necessarily form a closed subset.

The main purpose of this paper is to determine when the deformation space of such point configurations admits a complex structure. In the next section, we give reasonable conditions which enable us to conclude that such a deformation space admits a standard complex structure from certain related Teichmüller spaces. The proof of this main theorem (Theorem 2.8) will be demonstrated in Section 3.

Received by the editors August 18, 2016 and, in revised form, January 13, 2017.

2010 Mathematics Subject Classification. Primary 30F60; Secondary 32G15.

The first author was partially supported by Grants-in-Aid for Scientific Research (C) Grant No. 25400127.

The second author was partially supported by Grants-in-Aid for Scientific Research (C) Grant No. 15K04925. 
We consider a Kleinian group as another famous object inducing the holomorphic dynamics. The set of fixed points of its elements, instead of periodic ones, plays a fundamental role. To introduce the deformation space of such points, we should involve the group action with the set of fixed points of a Kleinian group. In Section 4, we investigate such a situation. However, since the theory of infinitely generated Kleinian groups is still in the cradle, we only give some discussions and typical examples for finitely generated Kleinian groups. Generalization to the infinitely generated case is possible to some extent, but we leave it for future study.

\section{Definition AND STATEMENT OF THEOREM}

In this section, let $R$ be a Riemann surface and $E=\left\{p_{k}\right\}_{k=1}^{\infty}$ an ordered countable set of an infinite number of distinct points on $R$. Set $R^{*}=R-\bar{E}$.

Definition 2.1. Let $\mathrm{QC}(E, R)$ be the set of all pairs $\left(E^{\prime}, f\right)$ of ordered countable sets $E^{\prime}=\left\{q_{k}\right\}_{k=1}^{\infty}$ of an infinite number of distinct points on a Riemann surface $R^{\prime}$ and quasiconformal homeomorphisms $f$ of $R$ onto $R^{\prime}$ that give order-preserving bijections of $E$ to $E^{\prime}$ in the sense that $f\left(p_{k}\right)=q_{k}$ for every $k$. We say that two points $\left(E_{1}, f_{1}\right)$ and $\left(E_{2}, f_{2}\right)$ of $\mathrm{QC}(E, R)$ are equivalent if there is a conformal homeomorphism $\phi$ of $f_{1}(R)$ onto $f_{2}(R)$ which gives an order-preserving bijection of $E_{1}$ to $E_{2}$. The essential Teichmüller space $T(E, R)$ of $E$ in $R$ is the set of all equivalence classes $\left[\left(E^{\prime}, f\right)\right]$ with $\left(E^{\prime}, f\right) \in \mathrm{QC}(E, R)$.

Remark 2.2. This definition is different from the Teichmüller space of a closed subset in the Riemann sphere introduced by Lieb [8]. See also [13. Our Teichmüller space turns out to be a certain quotient space of the Teichmüller space defined in their manner.

The natural metric on $T(E, R)$ can be defined the same as the Teichmüller distance on the Teichmüller space. We recall the definition of the Teichmüller space of a Riemann surface later.

Definition 2.3. We define the Teichmüller distance $d_{T}$ on $T(E, R)$ by

$$
d_{T}\left(\left[\left(E_{1}, f_{1}\right)\right],\left[\left(E_{2}, f_{2}\right)\right]\right)=\inf _{g} \log K_{g},
$$

where the infimum is taken over all quasiconformal homeomorphisms $g$ of $f_{1}(R)$ onto $f_{2}(R)$ which give order-preserving bijections of $E_{1}$ to $E_{2}$, and $K_{g}$ denotes the maximal dilatation of $g$.

A Riemann surface $S$ is hyperbolic (or more precisely, admits a hyperbolic metric) if it can be represented as the quotient space of the unit disk $\mathbb{D}=\{z \in \mathbb{C} \mid$ $|z|<1\}$ (considered as the hyperbolic plane) by a Fuchsian model $G_{S}$ of $S$. The limit set $\Lambda\left(G_{S}\right)$ is contained in $\partial_{\infty} \mathbb{D}=\{z \in \mathbb{C}|| z \mid=1\}$. We say that $G_{S}$ is of the first kind or of the second kind according to $\partial_{\infty} \mathbb{D}=\Lambda\left(G_{S}\right)$ or not. We call

$$
\partial_{\infty} S=\left(\partial_{\infty} \mathbb{D}-\Lambda\left(G_{S}\right)\right) / G_{S}
$$

the ideal boundary at infinity of $S$. Hence $G_{S}$ is of the first kind if and only if $\partial_{\infty} S$ is empty.

Since $E$ consists of an infinite number of points, every connected component $S$ of the exterior $R^{*}=R-\bar{E}$ of $E$ admits the hyperbolic metric. Hereafter, in this paper, we impose the following conditions on the closure $\bar{E}$ :

(1) $\bar{E}$ is compact and its 2-dimensional measure is zero. 
(2) Every connected component of $\bar{E}$ that is not a singleton is isolated from the other components.

We will show that, if a pair $(E, R)$ satisfies certain geometric conditions, then $T(E, R)$ admits a natural complex Banach manifold structure from those of Teichmüller spaces $T(S)$ for the components $S$ of $R^{*}$.

Definition 2.4. We say that a hyperbolic Riemann surface $S$ satisfies the lower bound condition if the injectivity radius at every point of $S$ is uniformly bounded away from zero except in horocyclic cusp neighborhoods of area one. We say that $S$ satisfies the upper bound condition if the injectivity radius at every point of an $S^{\prime}$ is uniformly bounded from above, where $S^{\prime}$ is a suitable subsurface of $S$ such that the inclusion map $S^{\prime} \rightarrow S$ induces a surjective homomorphism $\pi_{1}\left(S^{\prime}\right) \rightarrow \pi_{1}(S)$. We say that a pair $(E, R)$ satisfies the bounded geometry condition if each connected component $S$ of $R^{*}$ satisfies the lower and upper bound conditions and unless $R$ is hyperbolic with $\partial_{\infty} R \neq \emptyset$.

Remark 2.5. A hyperbolic Riemann surface $S$ satisfies the bounded geometry condition if $S$ satisfies the lower and upper bound conditions and if $\partial_{\infty} S=\emptyset$. This was given in previous papers to study the action of Teichmüller modular groups. See for example [12, Definition 2.2]. The above definition is an adaptation to the pair $(E, R)$.

Now we recall the definition of the Teichmüller space. The Teichmüller space $T(S)$ of a Riemann surface $S$ is the set of all equivalence classes $[f]$ of quasiconformal homeomorphisms $f$ of $S$. Here we say that two quasiconformal homeomorphisms $f_{1}$ and $f_{2}$ of $S$ are equivalent if there exists a conformal homeomorphism $h: f_{1}(S) \rightarrow$ $f_{2}(S)$ such that $f_{2}^{-1} \circ h \circ f_{1}$ is homotopic to the identity on $R$. The homotopy is considered to be relative to the ideal boundary at infinity $\partial_{\infty} S$.

A quasiconformal mapping class is the homotopy equivalence class $[g]$ of quasiconformal automorphisms $g$ of a Riemann surface $S$, and the quasiconformal mapping class group $\operatorname{MCG}(S)$ of $S$ is the group of all quasiconformal mapping classes of $S$. Here the homotopy is again considered to be relative to the ideal boundary at infinity. Every element $[g] \in \operatorname{MCG}(S)$ induces a biholomorphic automorphism $[g]_{*}$ of $T(S)$ by $[f] \mapsto\left[f \circ g^{-1}\right]$. Let Aut $(T(S))$ denote the group of all biholomorphic automorphisms of $T(S)$. Then we have a homomorphism $\iota_{T}: \operatorname{MCG}(S) \rightarrow \operatorname{Aut}(T(S))$ given by $[g] \mapsto[g]_{*}$, and we define the Teichmüller modular group for $S$ by $\operatorname{Mod}(S)=\iota_{T}(\operatorname{MCG}(S))$. It is known that the homomorphism $\iota_{T}$ is bijective and thus $\operatorname{Mod}(R)=\operatorname{Aut}(T(S))$ except for a few low dimensional cases.

For an arbitrary hyperbolic Riemann surface $S$, the pure mapping class group is the subgroup of the mapping class group that consists of all quasiconformal mapping classes that fix every topological end (see [5]), and the subgroup of the Teichmüller modular group corresponding to the pure mapping class group is denoted by $\operatorname{Mod}_{\partial}(S)$.

In our case, we need to consider another subgroup of $\operatorname{Mod}(S)$, which consists of all quasiconformal mapping classes that fix each point of the boundary of $S$ made by $\bar{E}$.

Definition 2.6. For a connected component $S$ of $R^{*}$, we call the subgroup of $\operatorname{Mod}(S)$ corresponding to the group of all quasiconformal mapping classes that fix every topological end of $S$ given by $\bar{E}$ and every point of $\partial_{\infty} S$ given by $\bar{E}$ the 
external Teichmüller modular group of $S$ with respect to $E$, which is denoted by $\operatorname{Mod}_{E}(S)$.

Remark 2.7. By condition (2) on $\bar{E}$, the meaning of a topological end of $S$ and a point of $\partial_{\infty} S$ given by $\bar{E}$ is clear. Indeed, a connected component of $\bar{E}$ that is a singleton corresponds to a topological end of $S$ and a connected component of $\bar{E}$ that is a continuum corresponds to a closed subset of $\partial_{\infty} S$. If we exclude the case that $R$ is hyperbolic with $\partial_{\infty} R \neq \emptyset$ as we do in our theorem, then the entire $\partial_{\infty} S$ comes from $\bar{E}$, which means that a sequence in $S$ tending to $\partial_{\infty} S$ always accumulates in $\bar{E}$. Note that unless $R^{*}$ is connected, $\partial_{\infty} S \neq \emptyset\left(G_{S}\right.$ is of the second kind) for every component $S$ of $R^{*}$.

We say that a subgroup $\Gamma \subset \operatorname{Mod}(S)$ acts at a point $p \in T(S)$ discontinuously if there exists a neighborhood $U$ of $p$ such that the number of elements $\gamma \in \Gamma$ satisfying $\gamma(U) \cap U \neq \emptyset$ is finite. This is equivalent to the condition that there exist no sequence of distinct elements $\left\{\gamma_{n}\right\} \subset \Gamma$ such that $\lim _{n \rightarrow \infty} d_{T}\left(\gamma_{n}(p), p\right)=0$. Here $d_{T}$ is the Teichmüller distance on $T(S)$.

Now we are ready to state our main theorem as follows.

Theorem 2.8. Let $\left\{S_{j}\right\}$ be the set of all components of the exterior $R^{*}=R-\bar{E}$ of a countable set $E$ of infinite number of distinct points on a Riemann surface $R$. Suppose that $\bar{E}$ satisfies conditions (1) and (2) and that the pair $(E, R)$ satisfies the bounded geometry condition. Then for every $S_{j}$, the external Teichmüller modular group $\operatorname{Mod}_{E}\left(S_{j}\right)$ acts on the Teichmüller space $T\left(S_{j}\right)$ discontinuously and fixedpoint freely. Hence the quotient space

$$
T_{E}\left(S_{j}\right):=T\left(S_{j}\right) / \operatorname{Mod}_{E}\left(S_{j}\right)
$$

admits a canonical complex Banach manifold structure. Moreover, $T(E, R)$ is homeomorphic to the direct product $\prod_{j} T_{E}\left(S_{j}\right)$ with the box topology. Hence $T(E, R)$ admits a complex Banach manifold structure modeled on the product Banach space endowed with the supremum norm.

Then we give the following definition.

Definition 2.9. We call the quotient space $T_{E}(S)=T(S) / \operatorname{Mod}_{E}(S)$ the embellished moduli space of $S$ with respect to $E$.

The proof of Theorem 2.8 will be given in the next section.

Example 2.10. Let $C$ be a Cantor set in $R=\widehat{\mathbb{C}}$. Then we can choose an ordered countable dense subset $E$ of $C$ consisting of distinct points. Suppose that $C$ has area 0 and $R^{*}=\widehat{\mathbb{C}}-C$ satisfies the bounded geometry condition. This is the case for the classical Cantor set on the interval $[0,1] \subset \mathbb{R}$. Since $R^{*}$ is connected, we have

$$
T(E, R)=T_{E}\left(R^{*}\right)=T\left(R^{*}\right) / \operatorname{Mod}_{\partial}\left(R^{*}\right),
$$

and it admits a natural complex Banach manifold structure.

Note that, for every simply connected component $S$ of $R^{*}=R-\bar{E}$, we have $\operatorname{Mod}_{E}(S)=\{\mathrm{id}\}$ and $T_{E}(S)=T(S)$. Then the following corollary is clear. 
Corollary 2.11. Suppose that the area of $\bar{E}$ is zero. If every component $S_{j}$ of $R^{*}=R-\bar{E}$ is simply connected, then we have

$$
T(E, R)=\prod_{j} T\left(S_{j}\right),
$$

and hence $T(E, R)$ admits a complex Banach manifold structure.

Example 2.12. Suppose that $\bar{E}$ is connected in $R=\widehat{\mathbb{C}}$ and of zero area. Then we have the representation of $T(E, R)$ as in Corollary 2.11. In particular, if $R=\widehat{\mathbb{C}}$ and $E$ is dense in $\mathbb{R}$, then $T(E, R)$ is the "two-sided" universal Teichmüller space.

\section{Proof of the MAIN THEOREM}

In this section, we prove Theorem 2.8. We use the following proposition to see that the external Teichmüller modular group acts discontinuously and fixed-point freely. This corresponds to the statement of the first part of Theorem 2.8

Proposition 3.1. Let $S$ be a hyperbolic Riemann surface that satisfies the lower and upper bound conditions. Then we have the following:

(1) Suppose that $\partial_{\infty} S \neq \emptyset$. If a subgroup $\Gamma \subset \operatorname{Mod}(S)$ fixes every point of $\partial_{\infty} S$, then $\Gamma$ acts on $T(S)$ discontinuously and fixed-point freely.

(2) Suppose that $\partial_{\infty} S=\emptyset$ and $S=R-\bar{E}$, where $R$ is a Riemann surface and $\bar{E}$ is a totally disconnected infinite compact subset of $R$. If a subgroup $\Gamma \subset \operatorname{Mod}(S)$ fixes every topological end of $S$ given by $\bar{E}$, then $\Gamma$ acts on $T(S)$ discontinuously and fixed-point freely.

Proof. First we prove statement (1). Suppose to the contrary that the action of $\Gamma$ on $T(S)$ is not discontinuous. Then we may assume that there is a sequence $\left\{\left[\gamma_{n}\right]\right\}_{n} \subset$ $\Gamma$ such that, for the base point $o=[\mathrm{id}] \in T(S)$, the orbit $\left[\gamma_{n}\right]_{*}(o)$ accumulates to $o$. This implies that the maximal dilatations $K_{\gamma_{n}}$ of the representatives $\gamma_{n}$, which are quasiconformal automorphisms of $S$, tend to 1 . Since each $\gamma_{n}$ fixes every point of $\partial_{\infty} S, \gamma_{n}$ converge locally uniformly to the identity by passing to a subsequence if necessary. However, by the lower and upper bound conditions on $S$ and by the assumption that $\gamma_{n}$ fixes every point of $\partial_{\infty} S$, we see that $K_{\gamma_{n}}$ are uniformly bounded away from 1. See 4 and 6]. (In these papers, the statement was given for the reduced Teichmüller space but the condition that every point of $\partial_{\infty} S$ is fixed can make it still valid for the ordinary Teichmüller space.) Thus we derive a contradiction to prove the claim.

We show that $\Gamma$ acts on $T(S)$ fixed-point freely. Suppose that there is a point $p$ in $T(S)$ that is fixed by an element $[\gamma]$ in $\Gamma$. Then on the Riemann surface corresponding to the fixed point $p$, the mapping class $[\gamma]$ is realized as a conformal automorphism. We may assume that $p$ is the base point in $T(R)$, namely, the corresponding Riemann surface is $S$. Since the conformal automorphism fixes every point of $\partial_{\infty} S$, it must be the identity. Thus we have the claim.

Next we prove statement (2). For an arbitrary quasiconformal deformation $S^{\prime}$ of $S=R-\bar{E}$, we may also assume that $S^{\prime}$ is $R^{\prime}-\overline{E^{\prime}}$ for some Riemann surface $R^{\prime}$ with a totally disconnected infinite compact subset $\overline{E^{\prime}}$ of $R^{\prime}$. This is because the quasiconformal deformation of $S$ can be extended to that of $R$ by the measurable Riemann mapping theorem applied to the Beltrami differential on $S$ extended to $R$ with zero on $\bar{E}$. Hence the situations are the same for all points on $T(S)$ and we may consider the problems on the base surface $S$. 
To prove that $\Gamma$ acts on $T(S)$ discontinuously, suppose to the contrary that there is a sequence of quasiconformal automorphisms $\gamma_{n}$ of $S$ with $K_{\gamma_{n}} \rightarrow 1$ that fix every topological end determined by $\bar{E}$. Since each end corresponds to a single point of the totally disconnected set $\bar{E}$, we see that every $\gamma_{n}$ extends to an automorphism of $R$ by setting the identity on $\bar{E}$. Then by Lemma 3.2 below, $\gamma_{n}$ are quasiconformal on $R$ with $K_{\gamma_{n}} \rightarrow 1$. Since they fix all points in $\bar{E}$, we can extract a subsequence that converges locally uniformly on $R$, and hence on $S$. However, by the lower and upper bound conditions on $S$ with $\partial_{\infty} S=\emptyset$, this is impossible as we have mentioned before.

To prove that $\Gamma$ acts on $T(S)$ fixed-point freely, suppose that there is a conformal automorphism of $S$ that fixes every topological end determined by $\bar{E}$. As before and again using Lemma 3.2, these conformal maps extend to $R$ by setting the identity on $\bar{E}$ as conformal automorphisms. However, since $\bar{E}$ accumulates in $R$, they must be the identity. This completes the proof.

To consider quasiconformal extension, we need the Rickman lemma in the argument above and also later. See [2, Lemma 2]. The following statement is slightly generalized so that it can be applied on Riemann surfaces.

Lemma 3.2. Let $S$ be a Riemann surface and $K \subset S$ a closed subset. Let $\varphi$ and $\Phi$ be homeomorphisms of $S$ onto another Riemann surface $S^{\prime}$. Suppose that $\varphi$ is quasiconformal on $S, \Phi$ is quasiconformal on $S-K$ and $\varphi=\Phi$ on $K$. Then $\Phi$ is quasiconformal on $S$ and $K_{\Phi}=\max \left\{K_{\Phi_{\left.\right|_{S-K}}}, K_{\varphi}\right\}$.

Proof. Fix a local parameter $z$ in a small neighborhood $U \subset S$ at $p \in K$ and a local parameter $w$ in $\varphi(U) \cup \Phi(U) \subset S^{\prime}$ at $\varphi(p)$. We may assume that $w \circ \varphi \circ z^{-1}(U)$ and $w \circ \Phi \circ z^{-1}(U)$ are bounded in $\mathbb{C}$. Then as is mentioned in 2, it is enough to show that $u=\operatorname{Re}\left(w \circ \Phi \circ z^{-1}-w \circ \varphi \circ z^{-1}\right)$ is locally $H^{1}$ near $p$. Here, multiplying a suitable cut-off function to $u$, we may assume that $u$ has a compact support in $U$. Then smoothing $u$ near $u=0$ as in the original proof, we can show that $u$ is locally $H^{1}$ near $p$.

Since the external Teichmüller modular group $\Gamma=\operatorname{Mod}_{E}\left(S_{j}\right)$ and the pair $(E, R)$ satisfy either conditions (1) or (2) in Proposition 3.1 under the assumptions of Theorem 2.8, we obtain the first part of this theorem. The second part follows from the next proposition.

Proposition 3.3. Let $\left\{S_{j}\right\}$ be the set of all components of the exterior $R^{*}=$ $R-\bar{E}$ of a countable set $E$ of an infinite number of distinct points on a Riemann surface $R$. Suppose that $\bar{E}$ satisfies conditions (1) and (2). There is a surjective homeomorphism

$$
\Pi: T(E, R) \rightarrow \prod_{j} T_{E}\left(S_{j}\right)
$$

Proof. First we define the map $\Pi$. Take a point $\left[\left(E^{\prime}, f\right)\right]$ of $T(E, R)$. Then for every component $S_{j}$ of $R^{*}=R-\bar{E}$, the restriction $f_{j}=\left.f\right|_{S_{j}}$ is a quasiconformal homeomorphism of $S_{j}$, and determines a point of $T\left(S_{j}\right)$. If $\left(E^{\prime}, f\right)$ is equivalent to $\left(E^{\prime \prime}, g\right)$, that is, if there is a conformal homeomorphism $\phi: f(R) \rightarrow g(R)$ such that $\phi \circ f=g$ on $\bar{E}$, then $g_{j}{ }^{-1} \circ \phi \circ f_{j}$ gives a quasiconformal automorphism of $S_{j}$ which fixes every topological end of $S_{j}$ given by $\bar{E}$ and every point of $\partial_{\infty} S_{j}$. 
Hence they induce the same point of $\prod_{j} T_{E}\left(S_{j}\right)$. Thus we have a well-defined map $\Pi: T(E, R) \rightarrow \prod_{j} T_{E}\left(S_{j}\right)$.

Next we show that $\Pi$ is bijective. Take a point $\left\{\left[\left(S_{j}^{\prime}, f_{j}\right)\right]\right\}_{j}$ of $\prod_{j} T_{E}\left(S_{j}\right)$. Then by the measurable Riemann mapping theorem, we can construct a quasiconformal homeomorphism $f: R \rightarrow R^{\prime}=f(R)$ such that $f \circ\left(f_{j}\right)^{-1}$ is conformal on $S_{j}^{\prime}$ for every $j$, which determines a point of $T(E, R)$. This shows that $\Pi$ is surjective.

Moreover, we show that $\Pi$ is injective, and that the above construction of the point of $T(E, R)$ gives the well-defined inverse $\Pi^{-1}$. Suppose that $\left\{\left[\left(S_{j}^{\prime}, f_{j}\right)\right]\right\}_{j}$ and $\left\{\left[\left(S_{j}^{\prime \prime}, g_{j}\right)\right]\right\}_{j}$ are the same point of $\prod_{j} T_{E}\left(S_{j}\right)$. Let $f: R \rightarrow R^{\prime}$ and $g: R \rightarrow R^{\prime \prime}$ be quasiconformal homeomorphisms constructed as above from $\left\{\left[\left(S_{j}^{\prime}, f_{j}\right)\right]\right\}_{j}$ and $\left\{\left[\left(S_{j}^{\prime \prime}, g_{j}\right)\right]\right\}_{j}$, respectively. Then by the definition, there is a conformal homeomorphism $\psi_{j}: f\left(S_{j}\right) \rightarrow g\left(S_{j}\right)$ such that $\psi_{j} \circ f=g$ on every topological end of $S_{j}$ determined by $\bar{E}$ and every point of $\partial_{\infty} S_{j}$ for each $j$. We consider $g^{-1} \circ \psi_{j} \circ f$, which fixes every topological end of $S_{j}$ determined by $\bar{E}$ and every point of $\partial_{\infty} S_{j}$.

Here we first use Lemma 3.4 below. It follows that $g^{-1} \circ \psi_{j} \circ f$ extends continuously to the relative boundary $\partial_{R} S_{j}$ of $S_{j}$ in $R$ by setting the identity on $\partial_{R} S_{j}$ for every $j$. In this way, we have an automorphism $g^{-1} \circ \psi \circ f$ of $R$ that is quasiconformal on $R^{*}$ and the identity on $\bar{E}$, where $\psi: R^{\prime} \rightarrow R^{\prime \prime}$ is a homeomorphism such that $\left.\psi\right|_{S_{j}}=\psi_{j}$. Then by Lemma 3.2, $g^{-1} \circ \psi \circ f$ is quasiconformal on $R$, and by the assumption that the area of $\bar{E}$ is zero, $\psi$ is conformal. Hence $f$ and $g$ determine the same point of $T(E, R)$.

Finally, we show that $\Pi$ and $\Pi^{-1}$ are continuous. Let $\left[\left(E_{n}, f_{n}\right)\right]$ be a sequence converging to some $\left[\left(E_{0}, f_{0}\right)\right]$ in $T(E, R)$. Then we can choose suitable representatives $f_{n}$ and $f_{0}$ so that the complex dilatations $\mu_{n}$ of $f_{n}$ converge to the complex dilatation $\mu_{0}$ of $f_{0}$ with respect to the supremum norm. The restrictions of $f_{n}$ and $f_{0}$ to each $S_{j}$ define the points of $T_{E}\left(S_{j}\right)$ and thus the images of $\Pi$. Since $\left.\mu_{n}\right|_{S_{j}}$ converges to $\left.\mu_{0}\right|_{S_{j}}$ in the supremum norm uniformly over $j$, we see that $\Pi\left[\left(E_{n}, f_{n}\right)\right]$ converges to $\Pi\left[\left(E_{0}, f_{0}\right)\right]$ in $\prod_{j} T_{E}\left(S_{j}\right)$. The continuity of $\Pi^{-1}$ is similarly obtained.

The following lemma is a slight generalization of a claim in [3, Proposition 2.3], in which they treated the case where $f: S \rightarrow S$ is homotopic to the identity relative to $\partial_{\infty} S$.

Lemma 3.4. Let $S$ be a connected component of the exterior $R^{*}=R-\bar{E}$ of a countable set $E$ of infinite number of distinct points on a Riemann surface $R$. Suppose that $\bar{E}$ satisfies conditions (1) and (2). Let $\partial_{R} S$ be the relative boundary of $S$ in $R$. Assume that there is a compact connected component $L$ of $\partial_{R} S$ that is not a singleton and a quasiconformal automorphism $f$ of $S$ that fixes every point of the part of $\partial_{\infty} S$ corresponding to $L$. Then $f$ extends continuously to $L$ by setting the identity on $L$.

Proof. Let $f$ be such a $K$-quasiconformal automorphism of $S$ and $\tilde{f}$ a lift of $f$ to the unit disk $\mathbb{D}$. We also have an interval $\widetilde{L}$ on $\partial_{\infty} \mathbb{D}$ that is a lift of the compact part of $\partial_{\infty} S$ corresponding to $L \subset \partial_{R} S$. Then we may assume that $\tilde{f}$ extends continuously to the identity on $\widetilde{L}$.

For any point $\xi \in L$, take a sequence of points $x_{n} \in S$ that converges to $\xi$. Lifting them to $\mathbb{D}$ and $\widetilde{L}$, we have a sequence $z_{n} \in \mathbb{D}$ that converges to an interior point $\zeta$ of the interval $\widetilde{L}$. Then we may assume that the visual measures of $\widetilde{L}$ viewed 
from $z_{n}$ are uniformly bounded away from zero. This implies that for a conformal automorphism $h_{n}$ of $\mathbb{D}$ sending $z_{n}$ to the origin 0 and for some three distinct points $a, b$ and $c$ on $\partial \mathbb{D}$, each conjugate $h_{n} \tilde{f} h_{n}^{-1}$ fixes $a, b$ and $c$. Since all $h_{n} \tilde{f} h_{n}^{-1}$ are $K$ quasiconformal, it has a subsequence converging uniformly to a $K$-quasiconformal automorphism of $\mathbb{D}$.

By this compactness property, we obtain that $\left|h_{n} \tilde{f} h_{n}^{-1}(0)\right|$ is uniformly bounded by some positive constant strictly less than 1 . Hence the hyperbolic distance $d_{\mathbb{D}}\left(z_{n}, \tilde{f}\left(z_{n}\right)\right)$ on $\mathbb{D}$, which coincides with the hyperbolic distance $d_{S}\left(x_{n}, f\left(x_{n}\right)\right)$ on $S$, is uniformly bounded by some constant. Since the ratio of the distance on $R$ to that of $S$ tends to zero as $x_{n}$ approaches $L$, we see that the distance $d_{R}\left(x_{n}, f\left(x_{n}\right)\right)$ on $R$ converges to zero. This shows that $f$ extends continuously to $\xi \in L$ by $f(\xi)=\xi$.

Remark 3.5. We expect that condition (2) on $\bar{E}$ can be removed from the assumption of Theorem 2.8. This condition is used in the above arguments to verify that a quasiconformal automorphism $f$ of $S$ fixing each topological end and each point of the boundary at infinity extends continuously to $\partial_{R} S$.

\section{Countable sets on $\widehat{\mathbb{C}}$ ordered by MöBius group aCtion}

An ordered countable subset $E$ in a Riemann surface $R$ is often obtained from a suitable group action. For the sake of simplicity, we discuss the case where $R=\widehat{\mathbb{C}}$ and the group $G$ is torsion-free and finitely generated, namely, it is generated by a finite number of ordered Möbius transformations $g_{1}, g_{2}, \ldots, g_{n}$.

We give a canonical order to $G$ by using the word length of a reduced word expression and, in addition, by giving the elements of same length the lexicographic order with respect to the ordered generators. Then we regard $G$ as an ordered set starting with

$$
e, g_{1}, g_{1}^{-1}, g_{2}, g_{2}^{-1}, \ldots, g_{n}, g_{n}^{-1}
$$

followed by those of length 2 , and so on. Here $e$ is the identity map.

Definition 4.1. Let $G$ be as above and let $\operatorname{Fix}(G)$ denote the set of all distinct fixed points of the elements of $G-\{e\}$. Set $E=\operatorname{Fix}(G)$ and define the canonical injection $\tau_{G}: E \rightarrow G$ uniquely so that every $z \in E$ is either the repelling fixed point or the single one of $\tau_{G}(z)$ according to $\tau_{G}(z)$ is either loxodromic or parabolic, and that $\tau_{G}(z)$ has the smallest order among all $g \in G-\{e\}$ satisfying this condition. We give an order to $E$ by $\tau_{G}$, which we call the order on $E=\operatorname{Fix}(G)$ induced from the $G$-action.

The fixed point set $E=\operatorname{Fix}(G)$ is clearly $G$-invariant in the sense that $g(E)=E$ as a set for every $g \in G$. Note that the canonical injection $\tau_{G}$ is not surjective. The following definition is conceptually the same as Definition 2.1 Concerning basic definitions and facts on Kleinian groups; see [1].

Definition 4.2. Let $G=\left\langle g_{1}, \ldots, g_{n}\right\rangle$ be a non-elementary torsion-free Kleinian group of the second kind generated by ordered Möbius transformations $g_{1}, g_{2}, \ldots, g_{n}$. For $E=\operatorname{Fix}(G)$, let $\mathrm{QC}(E, G)$ be the set of all pairs $\left(G^{\prime}, f\right)$. Here $G^{\prime}$ is a group generated by ordered Möbius transformations $g_{1}^{\prime}, g_{2}^{\prime}, \ldots, g_{n}^{\prime}$ such that the correspondence $g_{k} \mapsto g_{k}^{\prime}(k=1, \ldots, n)$ extends to an isomorphism $\theta: G \rightarrow G^{\prime}$, and $f$ is a quasiconformal automorphism of $\widehat{\mathbb{C}}$ that gives an order-preserving bijection of $E$ to $E^{\prime}=\operatorname{Fix}\left(G^{\prime}\right)$ equipped with the order induced from the $G^{\prime}$-action. We say 
that two points $\left(G_{1}, f_{1}\right)$ and $\left(G_{2}, f_{2}\right)$ of $\mathrm{QC}(E, G)$ are equivalent if there exists a Möbius transformation $\phi$ which gives an order-preserving bijection of $E_{1}=\operatorname{Fix}\left(G_{1}\right)$ to $E_{2}=\operatorname{Fix}\left(G_{2}\right)$. The essential Teichmüller space $T(E, G)$ of $E$ with $G$-action consists of all equivalence classes $\left[\left(G^{\prime}, f\right)\right]$ with $\left(G^{\prime}, f\right) \in \mathrm{QC}(E, G)$. The Teichmüller distance $d_{T}$ on $T(E, G)$ is defined similarly as before.

Concerning the isomorphism $\theta: G \rightarrow G^{\prime}$ in the above definition, we actually see the following property.

Proposition 4.3. For $\left(G^{\prime}, f\right) \in \mathrm{QC}(E, G)$, let $g_{k} \in G$ and $g_{k}^{\prime} \in G^{\prime}(k=1, \ldots, n)$ be generators as above. Then $f \circ g_{k}=g_{k}^{\prime} \circ f$ on $\bar{E}$ for every $k$. In particular, $\theta: G \rightarrow G^{\prime}$ is a type-preserving isomorphism induced by the conjugation of $f$ on the limit set $\Lambda(G)$.

Proof. First recall that every generator $g_{k}$ is determined by one or two fixed points of $g_{k}$, say $z_{k}$ (and $z_{k}^{*}$ ) in $E$, a distinct fixed point $z \in E$ of another element $g \in$ $G-\{e\}$, and $g_{k}(z)$ which is a fixed point of $g_{k} g g_{k}^{-1}$. Here we may assume that the canonical injection $\tau_{G}: E \rightarrow G$ satisfies $\tau_{G}\left(z_{k}\right)=g_{k}\left(\right.$ and $\tau_{G}\left(z_{k}^{*}\right)=g_{k}^{-1}$ ), $\tau_{G}(z)=g$, and $\tau_{G}\left(g_{k}(z)\right)=g_{k} g g_{k}^{-1}$. Then the images $f\left(z_{k}\right)$ (and $\left.f\left(z_{k}^{*}\right)\right), f(z)$, and $f\left(g_{k}(z)\right)$ are the corresponding fixed points of $g_{k}^{\prime}$ (and $\left.\left(g_{k}^{\prime}\right)^{-1}\right), g^{\prime}$, and $g_{k}^{\prime} g^{\prime}\left(g_{k}^{\prime}\right)^{-1}$, where $g^{\prime}=\theta(g)$. Hence they determine $g_{k}^{\prime}$ and, in particular, $f\left(g_{k}(z)\right)=g_{k}^{\prime}(f(z))$. Similarly, we see that $f\left(g_{k}(z)\right)=g_{k}^{\prime}(f(z))$ for every $z \in E$, and by the continuity, we obtain the first statement.

For a Kleinian group $G$, its limit set $\Lambda(G)$ coincides with $\bar{E}$. From the first statement, we have $f \circ g=\theta(g) \circ f$ on $\Lambda(G)$, that is, the isomorphism $\theta: G \rightarrow G^{\prime}$ is induced by $f$ on $\Lambda(G)$. Since $f$ maps the fixed point set of $g$ to that of $\theta(g), \theta$ must be type-preserving.

We see that $G^{\prime}$ is also a Kleinian group and, moreover, obtain the following claim. We define $A H(G)$ to be the algebraic deformation space consisting of all Möbius conjugacy classes of faithful discrete homomorphisms of $G$ into $\operatorname{PSL}(2, \mathbb{C})$ with the topology of elementwise convergence.

Lemma 4.4. There is a continuous injection

$$
\pi_{A}: T(E, G) \rightarrow A H(G)
$$

given by $\left[\left(G^{\prime}, f\right)\right] \mapsto[\theta]$, where $\theta$ is the type-preserving isomorphism of $G$ onto $G^{\prime}$ as above and $[\theta]$ is its Möbius conjugacy class.

Proof. For every $\left[\left(G^{\prime}, f\right)\right] \in T(E, G)$, the order-preserving bijection $f: E \rightarrow E^{\prime}$ induces the type-preserving isomorphism $\theta$ of $G$ onto $G^{\prime}$ by Proposition 4.3. Then we see that $G^{\prime}$ is discrete by the following lemma. Continuity of $\pi_{A}$ is a consequence from the continuous (in fact, holomorphic) dependence of quasiconformal homeomorphisms on Beltrami coefficients.

Injectivity of $\pi_{A}$ is seen as follows. Suppose that $\left[\left(G_{1}, f_{1}\right)\right]$ and $\left[\left(G_{2}, f_{2}\right)\right]$ in $T(E, G)$ define the isomorphisms $\theta_{1}: G \rightarrow G_{1}$ and $\theta_{2}: G \rightarrow G_{2}$ such that $\left[\theta_{1}\right]=\left[\theta_{2}\right]$ in $A H(G)$. By Proposition 4.3, $\theta_{i}$ is induced by the order-preserving bijection $f_{i}: \operatorname{Fix}(G) \rightarrow \operatorname{Fix}\left(G_{i}\right)(i=1,2)$. Then the Möbius transformation $\phi$ giving the conjugacy between $\theta_{1}$ and $\theta_{2}$ must be an order-preserving bijection of $\operatorname{Fix}\left(G_{1}\right)$ to $\operatorname{Fix}\left(G_{2}\right)$. This shows that $\left[\left(G_{1}, f_{1}\right)\right]=\left[\left(G_{2}, f_{2}\right)\right]$ by definition.

The following lemma was proved in [15, Lemma 2.8], which is due to the classification theorem by Sullivan [14. 
Lemma 4.5. Let $G$ be a subgroup of $\operatorname{PSL}(2, \mathbb{C})$ which contains no elliptic elements and fixes no points in $\widehat{\mathbb{C}}$. If the set of all fixed points of elements in $G-\{e\}$ is contained in a proper closed subset of $\widehat{\mathbb{C}}$, then $G$ is discrete.

The definition of our Teichmüller space would be more natural if we took the group equivariance of $f$ into account. However, such a space turns out to be the well-known quasiconformal deformation space $Q H(G)$ of $G$, namely, the totality of Möbius conjugacy classes of quasiconformal deformations of $G$. It is easy to see that there is a continuous injection,

$$
\iota_{Q}: Q H(G) \rightarrow T(E, G) .
$$

On the other hand, the natural inclusion map

$$
\iota: Q H(G) \rightarrow A H(G)
$$

is a continuous injection, and clearly $\pi_{A} \circ \iota_{Q}=\iota$.

Now let $S_{j}$ be a connected component of $\widehat{\mathbb{C}}-\bar{E}=\widehat{\mathbb{C}}-\Lambda(G)$ and $\operatorname{Stab}_{G}\left(S_{j}\right)$ the stabilizer subgroup of $G$ for $S_{j}$. Let $\operatorname{Mod}_{N}\left(S_{j}\right)$ be the subgroup of the external Teichmüller modular group $\operatorname{Mod}_{E}\left(S_{j}\right)$ consisting of all elements corresponding to "allowable" automorphisms $\phi_{j}$ of $S_{j}$, namely, such $\phi_{j}$ that $\phi_{j} \circ g=g \circ \phi_{j}$ for every $g \in \operatorname{Stab}_{G}\left(S_{j}\right)$. Then we can identify $\operatorname{Mod}_{N}\left(S_{j}\right)$ with a subgroup of $\operatorname{Mod}\left(R_{j}\right)$, which is denoted by $\operatorname{Mod}_{N}\left(R_{j}\right)$, where $R_{j}=S_{j} / \operatorname{Stab}_{G}\left(S_{j}\right)$.

The Ahlfors finiteness theorem asserts that for a finitely generated Kleinian group $G$ of the second kind, the region of discontinuity $\Omega(G)=\widehat{\mathbb{C}}-\Lambda(G)$ has finitely many non-equivalent components $\left\{S_{j}\right\}_{j=1}^{m}$ such that

$$
\Omega(G) / G=\bigcup_{j=1}^{m} S_{j} / \operatorname{Stab}_{G}\left(S_{j}\right)=\bigcup_{j=1}^{m} R_{j} .
$$

Moreover, each $R_{j}$ is an analytically finite Riemann surface. The structure theorem for the quasiconformal deformation space $Q H(G)$ for $G$ (see Kra [7]) says that $Q H(G)$ is homeomorphic to

$$
\prod_{j=1}^{m} T\left(R_{j}\right) / \operatorname{Mod}_{N}\left(R_{j}\right)
$$

and $\operatorname{Mod}_{N}\left(R_{j}\right)$ acts on $T\left(R_{j}\right)$ discontinuously and fixed-point freely. Hence $Q H(G)$ admits a finite dimensional complex structure.

Recall that this complex structure is natural in a sense that every element of $G$ moves holomorphically and that a system of points in $\operatorname{Fix}(G)$ gives holomorphic local coordinates. In particular, the natural map $\iota: Q H(G) \rightarrow A H(G)$ induces a complex structure on its injective image $\iota(Q H(G))$. Hence we obtain the following consequence.

Proposition 4.6. The image $\iota_{Q}(Q H(G))$ admits a finite dimensional (induced) complex structure, and $\pi_{A}: \iota_{Q}(Q H(G)) \rightarrow \iota(Q H(G))$ is a biholomorphic homeomorphism. In particular, $T(E, G)$ admits a finite dimensional complex structure when $T(E, G)=\iota_{Q}(Q H(G))$.

Remark 4.7. We know that $T(E, \widehat{\mathbb{C}})$ has a natural complex structure by Theorem 2.8 under a certain additional assumption on $\bar{E}=\Lambda(G)$. If $G$ is finitely generated and of the second kind, then the solution of the Ahlfors measure zero problem 
says that the 2-dimensional measure of $\Lambda(G)$ is zero. Hence condition (1) on $\bar{E}$ is satisfied in the present case; we have only to assume condition $(2)$. Then $T(E, \widehat{\mathbb{C}})$ is homeomorphic to the infinite product

$$
\prod_{j} T\left(S_{j}\right) / \operatorname{Mod}_{E}\left(S_{j}\right)
$$

This can be understood as the group-free version of the above expression of $Q H(G)$.

We expect that the assumption $T(E, G)=\iota_{Q}(Q H(G))$ in Proposition 4.6 always holds true. We first show such an example and provide the complex structure for $T(E, G)$.

Example 4.8. Let $G=\left\langle g_{1}, \ldots, g_{n}\right\rangle$ be a Schottky group, namely, a finitely generated purely loxodromic free discrete group with totally disconnected limit set, generated by ordered $n(\geq 2)$ Möbius transformations $g_{1}, \ldots, g_{n}$. Let $S=\Omega(G)=\widehat{\mathbb{C}}-\bar{E}$ and $R=S / G$. Then,

$$
Q H(G)=T(R) / \operatorname{Mod}_{N}(R)
$$

has a natural complex structure, which is so-called the Schottky space.

In this case, $G^{\prime}$ is free and purely loxodromic, and hence a Schottky group for every $\left[\left(G^{\prime}, f\right)\right] \in T(E, G)$. This implies that $\pi_{A}(T(E, G)) \subset \iota(Q H(G))$ in $A H(G)$. Since $\pi_{A} \iota^{\iota} Q=\iota$ and $\pi_{A}$ is injective by Lemma 4.4 we have $T(E, G)=\iota_{Q}(Q H(G))$. Hence Proposition 4.6 also gives that $T(E, G)$ has the natural complex structure.

We can generalize this example to geometrically finite Kleinian groups. First we prepare the following claim, which will also be used later for geometrically infinite case.

Lemma 4.9. Let $G$ and $G^{\prime}$ be non-elementary, finitely generated, torsion-free Kleinian groups having simply connected invariant components $S$ and $S^{\prime}$ of $\Omega(G)$ and $\Omega\left(G^{\prime}\right)$, respectively. Assume that there is a quasiconformal automorphism $f$ of $\widehat{\mathbb{C}}$ that induces a type-preserving isomorphism $\theta: G \rightarrow G^{\prime}$ by the conjugation on $\Lambda(G)$. Then there is another quasiconformal automorphism $\widehat{f}$ of $\widehat{\mathbb{C}}$ that coincides with $f$ on $\widehat{\mathbb{C}}-S$ and induces $\theta$ by the conjugation on $\bar{S}$.

Proof. Let $\varphi: \mathbb{D} \rightarrow S$ and $\varphi^{\prime}: \mathbb{D} \rightarrow S^{\prime}$ be the Riemann maps from the unit disk $\mathbb{D}$. Let $\Gamma$ and $\Gamma^{\prime}$ be Fuchsian models of $G$ and $G^{\prime}$ given by $\varphi$ and $\varphi^{\prime}$, respectively. The correspondence of the elements of $G\left(G^{\prime}\right)$ and $\Gamma\left(\Gamma^{\prime}\right)$ under the Riemann maps will be written without using explicit representation. Under this correspondence, the type-preserving isomorphism $\theta: G \rightarrow G^{\prime}$ induces a type-preserving isomorphism $\widetilde{\theta}: \Gamma \rightarrow \Gamma^{\prime}$. We consider a quasiconformal automorphism $h=\varphi^{\prime-1} \circ f \circ \varphi$ of $\mathbb{D}$ and its extension $\widehat{h}$ to $\partial_{\infty} \mathbb{D}$, which is quasisymmetric. First we will show that $\widehat{h}$ induces $\widetilde{\theta}$ by the conjugation on $\partial_{\infty} \mathbb{D}$.

Take any attracting fixed point $\xi \in \partial_{\infty} \mathbb{D}$ of a hyperbolic element $\gamma_{0} \in \Gamma$ such that the corresponding element $g_{0} \in G$ is loxodromic. Since such hyperbolic fixed points are dense in $\partial_{\infty} \mathbb{D}$, we have only to prove that $\widehat{h} \circ \gamma(\xi)=\gamma^{\prime} \circ \widehat{h}(\xi)$ for every $\gamma \in \Gamma$, where $\gamma^{\prime}=\widetilde{\theta}(\gamma)$. Note that $\gamma(\xi)$ is the attracting fixed point of a hyperbolic element $\gamma_{1}=\gamma \circ \gamma_{0} \circ \gamma^{-1} \in \Gamma$.

We consider an orbit $\xi_{n}=\gamma_{0}^{n}(z) \in \mathbb{D}(z \in \mathbb{D}, n \in \mathbb{N})$ converging to $\xi$. Then it is easy to see that $x_{n}=\varphi\left(\xi_{n}\right) \in S$ converges to the attracting fixed point $x \in \Lambda(G)$ of $g_{0}$. Let $g \in G$ be the loxodromic element corresponding to $\gamma$ and set 
$g_{1}=g \circ g_{0} \circ g^{-1} \in G$. By the assumption $f \circ g(x)=g^{\prime} \circ f(x)$ for $g^{\prime}=\theta(g) \in G^{\prime}$, we have

$$
\lim _{n \rightarrow \infty} f \circ g\left(x_{n}\right)=f \circ g(x)=g^{\prime} \circ f(x)=\lim _{n \rightarrow \infty} g^{\prime} \circ f\left(x_{n}\right) .
$$

Here $f \circ g(x)=g^{\prime} \circ f(x)$ is the attracting fixed point of $g_{1}^{\prime}=\theta\left(g_{1}\right) \in G^{\prime}$. We transport this situation to $\mathbb{D}$ by the Riemann maps. Then, via the attracting fixed point of $\gamma_{1}^{\prime}=\widetilde{\theta}\left(\gamma_{1}\right) \in \Gamma^{\prime}$, we can conclude that the following coincides

$$
\lim _{n \rightarrow \infty} h \circ \gamma\left(\xi_{n}\right)=\lim _{n \rightarrow \infty} \gamma^{\prime} \circ h\left(\xi_{n}\right) .
$$

This yields $\widehat{h} \circ \gamma(\xi)=\gamma^{\prime} \circ \widehat{h}(\xi)$, which we aim to show.

Next we extend the quasisymmetric automorphism $\widehat{h}$ of $\partial_{\infty} \mathbb{D}$ to a quasiconformal automorphism of $\mathbb{D}$ in the conformally natural way by the Douday-Earle extension ([1]). We denote this extension by the same symbol $\widehat{h}$. By the conformal naturality, this also induces $\widetilde{\theta}: \Gamma \rightarrow \Gamma^{\prime}$. Then we define a quasiconformal homeomorphism $\widehat{f}: S \rightarrow S^{\prime}$ by $\widehat{f}=\varphi^{\prime} \circ \widehat{h} \circ \varphi^{-1}$. Clearly, this induces $\theta: G \rightarrow G^{\prime}$ by the conjugation on $S$.

Finally, we verify that $\widehat{f}$ extends to $\partial_{\widehat{\mathbb{C}}} S$ as a homeomorphism and coincides with $f$ on $\partial_{\widehat{\mathbb{C}}} S$. Then we obtain an automorphism of $\widehat{\mathbb{C}}$ denoted by the same $\widehat{f}$ by setting $\widehat{f}=f$ on $\widehat{\mathbb{C}}-S$, which turns out to be quasiconformal by Lemma 3.2. By the construction, $\widehat{f}$ coincides with $f$ on the ideal boundary at infinity of $S$, which means in our case that $\widehat{h}$ and $h$ on $\mathbb{D}$ extend continuously to $\partial_{\infty} \mathbb{D}$ having the same boundary map. Then by the original claim of Lemma 3.4 as in [3, Proposition 2.3], we see that $\widehat{f}$ extends to $\partial_{\widehat{\mathbb{C}}} S$ continuously and coincides with $f$ on $\partial_{\widehat{\mathbb{C}}} S$.

By using Lemma 4.9, we have the following.

Proposition 4.10. If $G$ is a non-elementary, geometrically finite, torsion-free Kleinian group of the second kind, then $T(E, G)=\iota_{Q}(Q H(G))$ and $T(E, G)$ has the natural complex structure.

Proof. For every $\left[\left(G^{\prime}, f\right)\right] \in T(E, G)$, we consider the type-preserving isomorphism $\theta: G \rightarrow G^{\prime}$. Take any component $S$ of $\Omega(G)$ and $\operatorname{Stab}_{G}(S)$. Then it is easy to see that $S^{\prime}=f(S)$ is a component of $G^{\prime}$ and the restriction of $\theta$ to $\operatorname{Stab}_{G}(S)$ is a type-preserving isomorphism $\theta: \operatorname{Stab}_{G}(S) \rightarrow \operatorname{Stab}_{G}^{\prime}\left(S^{\prime}\right)$. Note that $H=\operatorname{Stab}_{G}(S)$ and $H^{\prime}=\operatorname{Stab}_{G}^{\prime}\left(S^{\prime}\right)$ are function groups with the invariant components $S$ and $S^{\prime}$ and with the limit sets $\Lambda(H)=\partial_{\widehat{\mathbb{C}}} S$ and $\Lambda\left(H^{\prime}\right)=\partial_{\widehat{\mathbb{C}}} S^{\prime}$, respectively.

We apply the Maskit theorem ([10]) concerning a condition under which the typepreserving isomorphism between function groups is induced geometrically by an orientation-preserving homeomorphism, in fact, a quasiconformal homeomorphism between the invariant components. In our case, we have only to check that the restriction of the type-preserving isomorphism $\theta: H \rightarrow H^{\prime}$ to every non-elementary factor subgroup $J \subset H$ is orientation-preserving. Note that such a factor subgroup $J$ has the simply connected invariant component of $\Omega(J)$ containing $S$. By Lemma 4.9, $\left.\theta\right|_{J}$ is induced by a quasiconformal homeomorphism of the invariant component and, in particular, it is type-preserving. Then we can apply the Maskit theorem to conclude that $\left.\theta\right|_{H}$ is induced by a quasiconformal homeomorphism $\widehat{f}_{S}: S \rightarrow S^{\prime}$.

We choose such quasiconformal homeomorphisms $\widehat{f}_{S}$ for all non-equivalent components $S$ of $\Omega(G)$ and extend them to $\Omega(G)$ in the $G$-equivariant way. Then we obtain a quasiconformal homeomorphism $\widehat{f}: \Omega(G) \rightarrow \Omega\left(G^{\prime}\right)$ that induces $\theta$ by the 
conjugation. Since $G$ is geometrically finite, the Marden isomorphism theorem ([9]) implies that $\widehat{f}$ extends further to $\widehat{\mathbb{C}}$ quasiconformally and induces $\theta$. (As a result, we see that $\widehat{f}$ coincides with $f$ on $\Lambda(G)$.) This implies $[\theta] \in Q H(G)$ and hence $\pi_{A}\left(\left[\left(G^{\prime}, f\right)\right]\right)=\iota([\theta])$. Since $\pi_{A} \circ \iota_{Q}=\iota$ and $\pi_{A}$ is injective by Lemma 4.4 we have $\left[\left(G^{\prime}, f\right)\right] \in \iota_{Q}(Q H(G))$ and thus $T(E, G)=\iota_{Q}(Q H(G))$.

Finally, the following proposition shows that we can also deal with a certain case where $G$ is not necessarily geometrically finite.

Proposition 4.11. Let $G$ be a non-elementary, finitely generated, torsion-free Kleinian group of the second kind such that every component of $\Omega(G)$ is simply connected. Then $T(E, G)=\iota_{Q}(Q H(G))$ and $T(E, G)$ has the natural complex structure.

Proof. We can prove this claim similarly to Proposition 4.10. The setting is the same and we use the same notation. For any simply connected component $S$ of $\Omega(G)$, we choose a quasiconformal homeomorphism $\widehat{f}_{S}: S \rightarrow S^{\prime}$ that induces the type-preserving isomorphism $\theta: G \rightarrow G^{\prime}$ restricted to $\operatorname{Stab}_{G}(S)$. In the present case, $\widehat{f}_{S}$ is given by applying Lemma 4.9. Then we can assume that $\widehat{f}_{S}$ already extends continuously to $\partial_{\widehat{\mathbb{C}}} S$. We replace the original $f$ with $\widehat{f}_{S}$ on $S$, and perform the same operation on all components $S$ of $\Omega(G)$ in the $G$-equivariant way. The resulting automorphism $\widehat{f}$ of $\widehat{\mathbb{C}}$ is quasiconformal by Lemma 3.2. This can verify the statement of this proposition in the same way as before.

\section{REFERENCES}

[1] A. Douady and C. J. Earle, Conformally natural extension of homeomorphisms of the circle, Acta Math. 157 (1986), no. 1-2, 23-48, DOI 10.1007/BF02392590. MR857678

[2] A. Douady and J. H. Hubbard, On the dynamics of polynomial-like mappings, Ann. Sci. École Norm. Sup. (4) 18 (1985), no. 2, 287-343. MR816367

[3] C. J. Earle and C. McMullen, Quasiconformal isotopies, Holomorphic functions and moduli, Vol. I (Berkeley, CA, 1986), Math. Sci. Res. Inst. Publ., vol. 10, Springer, New York, 1988, pp. 143-154, DOI 10.1007/978-1-4613-9602-4_12. MR955816

[4] E. Fujikawa, Modular groups acting on infinite dimensional Teichmüller spaces, In the tradition of Ahlfors and Bers, III, Contemp. Math., vol. 355, Amer. Math. Soc., Providence, RI, 2004, pp. 239-253, DOI 10.1090/conm/355/06455. MR2145066

[5] E. Fujikawa, Pure mapping class group acting on Teichmüller space, Conform. Geom. Dyn. 12 (2008), 227-239, DOI 10.1090/S1088-4173-08-00188-4. MR2466018

[6] E. Fujikawa, H. Shiga, and M. Taniguchi, On the action of the mapping class group for Riemann surfaces of infinite type, J. Math. Soc. Japan 56 (2004), no. 4, 1069-1086, DOI 10.2969/jmsj/1190905449. MR2091417

[7] I. Kra, On spaces of Kleinian groups, Comment. Math. Helv. 47 (1972), 53-69, DOI 10.1007/BF02566788. MR0306485

[8] G. S. Lieb, Holomorphic motions and Teichmuller space, ProQuest LLC, Ann Arbor, MI, 1989. Thesis (Ph.D.)-Cornell University. MR2638376

[9] A. Marden, The geometry of finitely generated Kleinian groups, Ann. of Math. (2) 99 (1974), 383-462, DOI 10.2307/1971059. MR0349992

[10] B. Maskit, Isomorphisms of function groups, J. Analyse Math. 32 (1977), 63-82, DOI 10.1007/BF02803575. MR0463430

[11] K. Matsuzaki and M. Taniguchi, Hyperbolic manifolds and Kleinian groups, Oxford Mathematical Monographs, The Clarendon Press, Oxford University Press, New York, 1998. Oxford Science Publications. MR 1638795

[12] K. Matsuzaki, Infinite-dimensional Teichmüller spaces and modular groups, Handbook of Teichmüller theory. Vol. IV, IRMA Lect. Math. Theor. Phys., vol. 19, Eur. Math. Soc., Zürich, 2014, pp. 681-716, DOI 10.4171/117-1/16. MR3289713 
[13] S. Mitra, Teichmüller contraction in the Teichmüller space of a closed set in the sphere, Israel J. Math. 125 (2001), 45-51, DOI 10.1007/BF02773373. MR1853804

[14] D. Sullivan, Quasiconformal homeomorphisms and dynamics. II. Structural stability implies hyperbolicity for Kleinian groups, Acta Math. 155 (1985), no. 3-4, 243-260, DOI 10.1007/BF02392543. MR806415

[15] M. Taniguchi, Teichmüller space of a countable set of points on the Riemann sphere, Filomat, to appear.

Department of Mathematics, Chiba University, Inage-ku, Chiba 263-8522, Japan

E-mail address: fujikawa@math.s.chiba-u.ac.jp

Department of Mathematics, Nara Women's University, Nara 630-8506, Japan

E-mail address: tanig@cc.nara-wu.ac.jp 